\title{
A Comparison between two Modified NSGA-II Algorithms for Solving the Multi-objective Flexible Job Shop Scheduling Problem
}

\author{
Aydin Teymourifar ${ }^{1,2, *}$, Gurkan Ozturk ${ }^{1,2}$, Ozan Bahadir $^{1,2}$ \\ ${ }^{1}$ Faculty of Engineering, Anadolu University, 26555, Eskisehir, Turkey \\ ${ }^{2}$ Computational Intelligence and Optimization Laboratory (CIOL), Anadolu University, 26555, Eskisehir, Turkey
}

Copyright (C) 2018 by authors, all rights reserved. Authors agree that this article remains permanently open access under the terms of the Creative Commons Attribution License 4.0 International License

\begin{abstract}
Many evolutionary algorithms have been used to solve multi-objective scheduling problems. NSGA-II is one of them that is based on the Pareto optimality concept and generally obtains good results. However, it is possible to improve its performance with some modifications. In this paper, two modified NSGA-II algorithms have been suggested for solving the multi-objective flexible job shop scheduling problem. The neighborhood structures defined for the problem are integrated into the algorithms to create better generations during the iterations. Also, their initial populations are created with an effective heuristic. In the first modified NSGA-II, after the creation of the offspring population, a neighbor of each individual in the parent population is constructed, and then one of them is selected according to the domination state of the solutions. Then the populations are merged to create a new population. In the second modified NSGA-II, only the solutions on the first and second fronts of the parent population and also their neighbors are merged with the offspring population. Other operators of the algorithms like the non-dominated sorting and calculating the crowding distances are as the classic NSGA-II. A comparison is done with a classic NSGA-II based on two metrics. The results show that as it is in the first modified NSGA-II, including neighbors of more individuals of the population provides better results because it increases diversity and intensity of the search. The performance of the second modified NSGA-II is almost similar to the NSGA-II. So, it can be concluded that although integrating the neighborhood structures can improve the performance of search, it is better to define that the structures should be applied to how many and which solutions, in otherwise the quality of search may not increase.
\end{abstract}

Keywords Flexible Job Shop Scheduling, Multi-objective Optimization, NSGA-II, Neighborhood Structures, Hybrid Algorithms

\section{Introduction}

Flexible job shop scheduling problem (FJSSP) is an NP-hard problem that has been investigated by many researchers in the recent decades. Many of the scheduling problems in the manufacturing and computer science can be modelled based on this problem. For example, Ho et al. have defined the production process of the printed circuit board (PCB) in the electronic manufacturing service (EMS) as a FJSSP [19]. Because of the complex structure of this combinatorial problem, it is difficult to solve it with the non-hybrid and exact methods [45]. So, several hybrid methods, which integrate the population-based meta-heuristics (P-metaheuristics), single-based meta-heuristics (S-metaheuristics), heuristics, mathematical programming, constraint programming, machine learning and graph-based methods are suggested for solving this problem [1][36][39][41][42][43][44][47][50]. Also, in many real scheduling problems of the manufacturing systems, it is necessary to deal with more than one objective. Therefore, the algorithms for the multi-objective optimization are widely researched in the scheduling literature. Generally, in these algorithms, the diversity and intensity of the search are increased to create better generations. There are many approaches for this aim. For example, Knowles and Corne have proposed the Pareto archived evolution strategy, in which a child is generated from each parent. If the child dominates the parent, it is selected. If the parent dominates the child, a new child is created by the 
mutation operator. If none of them dominates the other, one of them is chosen according to the diversity criteria [28]. The aim of this study is to propose two hybrid algorithms based on the non-dominated sorting genetic algorithm-II (NSGA-II) for solving the multi-objective FJSSP (MO-FJSSP). We attempt to intensify the search using effective neighborhood structures, integrated into the algorithms. In the first modified NSGA-II (M1-NSGA-II), like the classic NSGA-II, during the iterations, the offspring population is generated by applying the crossover and mutation operators into the parent population. After this, a neighbor is generated for each individual in the parent population and one of them is selected according to the domination state of them. Then, the offspring and the revised parent populations are merged to generate a new population. In the second modified NSGA-II (M2-NSGA-II), only the solutions in the first and second fronts of the parent population and their neighbors are merged with the offspring population. The initial populations of both algorithms are created based on the left shifts heuristic [43]. The algorithms are compared with a classic NSGA-II, whose initial population is created randomly, based on different metrics.

The next sections of this paper are arranged such that in the second section, the problem definition and the test instances are described. Previous studies are explained in the third section regarding the multi-objective concepts. Design and implementation steps of the algorithms are described in the fourth section. Then, the results and comparison are presented. Conclusion and future works are the last section of the paper.

\section{Problem Definition and Test Instances}

The assumptions about the model are (i) each job has one or more operations, (ii) assignable machine and processing time of the operations on them are predefined, (iii) the jobs are independent of each other, (iv) interruption during the process of the operations isn't allowed, (v) each machine can process at most one operation at any time, (vi) setting time of the machines are negligible, (vii) each machine can work independently from the other machines with maximum performance and there aren't maintenance and breakdown times [5][20][52].

Two general phases of the FJSSP are (i) assignment and (ii) sequencing. Because of the flexibility, in spite of the job shop scheduling problem (JSSP), the jobs can be processed on more than one route. Thus, in addition to the sequencing of operations on machines, it is necessary to make a decision for assigning them to the machines. According to these steps of the FJSSP, two approaches can be applied to solve the problem as (i) the hierarchical approach with decomposition and (ii) the integrated approach. In the first approach, assignment and sequencing steps are done sequentially [7][27][46][48], while in the second approach they are done simultaneously [12][18][22]. The hierarchical approach is based on the Brandimarte's decomposing method and decreases the complexity of problem [49].

Some of the used notations to represent the jobs, machines and their features are summarized in Table 1.

Table 1. The used notations

\begin{tabular}{|c||l|}
\hline Notation & Description \\
\hline \hline$n$ & Total number of the jobs \\
\hline$m$ & Total number of the machines \\
\hline$n_{i}$ & Total operation number of job $i$ \\
\hline$O_{i j}$ & The $j$-th operation of job $i$ \\
\hline$P_{i j k}$ & Processing time of $O_{i j}$ on machine $k$ \\
\hline$S T_{i j k}$ & Start time of $O_{i j}$ on machine $k$ \\
\hline$P J\left[O_{i j}\right]$ & Previous operation of $O_{i j}$ on the related job \\
\hline$P J\left[O_{i j}\right]$ & Successive operation of $O_{i j}$ on the related job \\
\hline$P M\left[O_{i j}\right]$ & Previous operation of the $O_{i j}$ on the assigned machine \\
\hline$S M\left[O_{i j}\right]$ & Successive operation of the $O_{i j}$ on the assigned machine \\
\hline$C_{m a x}$ & Maximal completion time of the machines (makespan) \\
\hline$W_{\max }$ & Maximal machine workload \\
\hline$W_{T}$ & Total workload of the machines \\
\hline
\end{tabular}

The objective function of the problem is defined as Equation 1 [52]. 


$$
\text { Minimize } f(x)=\left(C_{\max }, W_{\max }, W_{T}\right)
$$

The sequence constraint of operations can be denoted as Equation 2. According to this constraint, the difference between the start time of two consecutive operations of a job must be at least as much as the processing time of the preceding operation. In other words, each operation can only start after the predecessor one is finished.

$$
\text { s.t. } S T_{i j l}-S T_{i(j-1) k} \geq P_{i(j-1) k}, j=2, \ldots, n_{i} ; \quad \forall i, j, k
$$

Each machine can only process one operation at a time, and also an operation cannot leave the machine until it is finished. Therefore, the difference between the start time of two consecutive processes on a machine must be at least as much as the processing time of the preceding one. This is satisfied with the resource constraint, which is expressed as Equation 3.

$$
\begin{array}{r}
{\left[\left(S T_{l m k}-S T_{i j k}-P_{i j k} \geq 0\right)\right]} \\
o r \\
{\left[\left(S T_{i j k}-S T_{l m k}-P_{l m k} \geq 0\right)\right] ;} \\
\forall(i, j),(l, m), k
\end{array}
$$

It is clear that according to the order of operations on a machine, only one of these inequalities is valid. The first one is valid if $O_{i j}$ begins before $O_{l m}$ on machine $k$, and in otherwise the second one is valid.

In the literature, there are several test instances for the FJSSP like the Behnke and Geiger [4], Brandimarte [7], Hurink et al. [22], Dauzère-Pérès and Paulli [11], Chambers and Barnes [8], Kacem et al. [26], Fattahi et al. [17] instances. If all machines of a problem can process all of the operations that is a FJSSP with total flexibility, while in the partial FJSSP, each operation can be processed on a subset of the machines set [26][49]. The partial FJSSPs are generally more difficult than the total FJSSPs [3].

\section{Previous Studies}

In this section, the defined neighborhoods for the problem, some of the proposed multi-objective evolutionary algorithms and related concepts are summarized.

\subsection{Neighborhood structures for the FJSSP}

Several neighborhood structures are defined for the FJSSP in the literature, which are generally described with the corresponding disjunctive graph (DG) of a schedule. The structure $N_{1}$ creates neighbor $S^{\prime}$ of solution $S$ with permuting operations $O_{i j}$ and $O_{k l}$ that are successive on a critical block. In the structure $N_{2}$, the rule to select operations is similar to the structure $N_{1}$. However, in $N_{2}$, at least one of operations $O_{i j}, O_{k l}, \mathrm{PM}\left[O_{i j}\right]$ and $\mathrm{SM}\left[O_{k l}\right]$ is not on a critical path. In otherwise, applying a swap on them can't change the makespan. Reversing operations $O_{i j}, O_{k l}, \mathrm{SJ}\left[O_{i j}\right], \mathrm{SM}\left[\mathrm{SJ}\left[O_{k l}\right]\right]$, PM[PJ[$\left.\left.O_{k l}\right]\right]$ and $\operatorname{PJ}\left[O_{k l}\right]$ should be also checked to achieve a shorter critical path. In the structure $N_{3}$, randomly a machine and a job that is not assigned to the machine are chosen. Then the job is transferred to another valid position on the machine. In the neighborhood structure $N_{4}$, all operations of a critical block are moved to the very beginning or very end of the block. Nowicki and Smutnicki have proposed the neighborhood structure $N_{5}$ that is smaller than the structures $N_{1}$ and $N_{4}$ and searches on the borders of a block. However, in this structure, one critical block is selected randomly and the search is done on it. The neighborhood structure $N_{6}$ switches schedule $S$ to neighbor $S^{\prime}$ with interchanging operations $O_{i j}$ and $O_{k l}$, which are on a same critical block [2][6][16][33][34].

\subsection{Multi-Objective Scheduling}

Genotype corresponds to the sequence of the genes in the chromosomes and phenotypes are the observable characteristics of the sequences. In the multi-objective scheduling, the phenotypes are the values of the objective function and the genotypes are the values of the variables. There are two approaches for extracting the phenotypes from the genotypes, (i) the prior approach and (ii) the posterior approach. In the first approach, the preference information of decision maker is defined previously and the multi-objective problem transforms to a single-objective problem with a linear weighted summation of the objective functions. For example, if $f_{1}, f_{2}, \ldots, f_{n}$ is the vector that represents an objective function then in a prior approach, these criteria transform to a weighted function as $f=\sum_{i=1}^{n} w_{i} f_{i}, 0 \leq w_{i} \leq 1, \sum_{i=1}^{n} w_{i}=1$. Based on a similar approach, some evolutionary algorithms 
assign a weight to each objective function and search in one direction. But, this approach has some shortcomings; for example, as seen in Figure 1, with a weight vector as $w^{\prime}=(0.5,0.5)$ for $f_{1}$ and $f_{2}$ objective functions, it is possible to find $B$ and $C$ Pareto optimal solutions easily but finding $A$ and $D$ points is difficult [10][31].

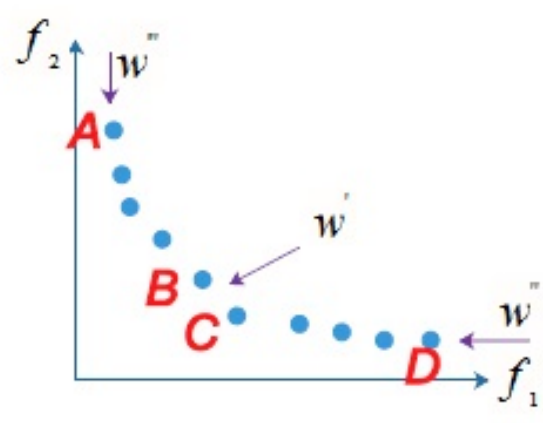

Figure 1. The multiple weight vector approach

The multiple weight vector can be applied to overcome this deficiency, because in this case, the search is done in different directions. There are many methods to apply this approach in the literature, (i) the Schaffer's method, in which, the population is divided into $M$ sub-populations, then it is adapted to $M$ fitness functions as $f_{1}(x), f_{2}(x), \ldots, f_{M}(x)$ [37]; (ii) the Kursawe's method that is similar to the Schaffer's method, while a probability is assigned to each objective function to choose a specific direction to search on it [23][30] and (iii) the Ishibuchi and Murata's method, in which weights of the objective functions are selected randomly [31].

The posterior approach is generally more preferred, where, the preference information of decision maker is not known in advance and the appropriate solutions are selected from the obtained ones after the search. In this approach, the Pareto optimality and the domination concepts are used. Solution $S^{1}$ dominates solution $S^{2}$ (denoted as $S^{1} \succ S^{2}$ ), if and only if $\left(\forall i \in M: f_{i}\left(S^{1}\right) \leq f_{i}\left(S^{2}\right)\right) \curlywedge\left(\exists i \in M: f_{i}\left(S^{1}\right)<f_{i}\left(S^{2}\right)\right)$ and $S^{1}$ is a Pareto optimal solution, if and only if $\neg \exists S^{2}: S^{2} \succ S^{1}$. If set $P_{a s}$ contains all of the Pareto optimal solutions as $P_{a s}=\left\{S^{1} \mid \neg \exists S^{2}: S^{2} \succ S^{1}\right\}$ that is the Pareto front. The set of corresponding values of the objective functions in $P_{a s}$ as $P_{F}=\left\{\mathbf{f}(s)=\left(f_{0}(s), f_{1}(s), \ldots, f_{m-1}(a s)\right) \mid z \in P_{a s}\right\}$ is the set of Pareto optimal front. If $P_{a s}$ is infinite, it is difficult to find it with finite solutions, so a subset of the set can be desired. The intended subset must be a good estimation of the Pareto front with a good diversity [10][21][51].

There are many evolutionary algorithms in the literature based on the Pareto optimality concept. The non-dominated sorting genetic algorithm (NSGA) and the NSGA-II are between the most famous of them based on the Pareto optimality concept. The NSGA that is presented by Deb and Srinivas has some shortcomings as (i) the high complexity, of the non-dominated sorting, which is $O\left(m N^{3}\right)$ at each iteration, (ii) the lack of elitism and (iii) the diversity adjustment requirement [13][15][35][54].

The NSGA-III algorithm, proposed by Deb and Jian in 2014, is similar to the NSGA-II but uses different selection operators. It begins with determining the reference points $(\mathrm{H})$ to place on the hyperplane. This also provides the diversity of the obtained solutions. This reference-point-based many-objective algorithm emphasizes population members that are non-dominated, yet close to a set of supplied reference points. The many-objective optimization problems contain four or more objectives. Twoobjective and three-objective problems fall into a different class as the resulting Pareto-optimal front. Mostly, they can be comprehensively visualized by graphical means. But a strict upper bound on the number of objectives for a many-objective optimization problem is not so clear, except for a few occasions, most practitioners are interested in a maximum of 10-15 objectives. Ciro et al., have compared the NSGA-II and NSGA-III algorithms for solving an open shop scheduling problem with resource constraints to minimize the total flow time and balancing the resources workload. Both of them have a similar performance, however, the NSGA-III has a slight higher hyper-volume average than the NSGA-II. In addition, the NSGA-III has better results for the large-sized benchmarks and also for those that have more than 2 objectives. This is because of the process of defining a hyperplane and some reference points to classify the solutions and also for selecting the best qualified for the next population [9][14][32].

The NSGA-II algorithm uses the fast non-dominated sorting, whose complexity is $O\left(m N^{2}\right)$. In this algorithm, the density is estimated by the crowding distance calculation, which causes to choose qualified solutions and its complexity is $O(m N \log N)$. The crowded comparison operator is an order operator that guides the selection process to the uniform distribution in the Pareto 
front. The comparison performs according to the non-dominated rank $\left(i_{\text {rank }}\right)$ and the local crowding distance $\left(i_{\text {distance }}\right)$, whose complexity is $O(2 N \log (2 N))$.

Several metrics are used for quantifying the performance of the multi-objective evolutionary algorithms. For example, there are some criteria to measure the accuracy, the coverage and the variance. The accuracy shows the closeness between the generated non-dominated and the best known solutions. The coverage indicates the diversity of solutions, while the variance represents the maximum range covered by a solution in the non-dominated front. There are various metrics in the literature to measure the diversity. The front spread $(F S)$ that is proposed by Zitzler, is a suitable indicator of the diversity. The large values of this metric are desired [53]. In addition to the diversity, it is important to know that how many points are in the non-dominated solutions set. The front occupation $(F O)$ is a measure suggested by Van Veldhuizen, which is defined in Equation 4 and its large values are desired [53].

$$
F O=\left|F_{1}\right|
$$

$\left|F_{1}\right|$ is the number of solutions on the Pareto front. $D$ is another measure proposed by Van Veldhuizen whose small values are desired. This measure that is defined in Equation 5, can't properly represent the diversity because it indicates the average distance between the achieved solutions from the Pareto front [53].

$$
D=\frac{1}{\left|F_{1}\right|} \sum_{S} d\left(S^{1}, S^{2}\right)
$$

$d\left(S^{1}, S^{2}\right)$ are the distance between solutions $S^{1}$ and $S^{2}$ according to the objective functions $\left(f_{i}\right)$ in the Euclidean distance system, which are calculated as Equation 6 [53], where $m$ is the number of objectives.

$$
d\left(S^{1}, S^{2}\right)=\sqrt{\sum_{i=0}^{m-1}\left(f_{i}\left(S^{2}\right)-f_{i}\left(S^{1}\right)\right)^{2}}
$$

In the NSGA-II, the $\triangle$ measure is used to evaluate the distribution of the points in the first front of the Pareto solutions. This measure is defined as Equation 7 [13][15].

$$
\Delta=\sum_{i=1}^{\left|F_{1}\right|} \frac{\left|d_{i}-\bar{d}\right|}{\left|F_{1}\right|}
$$

$d_{i}$ is the distance between two successive solutions in the Pareto front as it is defined in Equation 6 and $\bar{d}$ is the average of them, which is calculated for the last iteration. After 10 iterations, the average and variance of the $\triangle$ are calculated for comparison [13][15].

Tavakkoli et al. have designed an algorithm, which is compared with the NSGA-II, according to (i) a quality metric, (ii) a spacing metric and (iii) a diversification metric. Non-dominated solutions of the algorithms are put together and their ratio is calculated with the quality metric. Spacing metric that is defined as Equation 8, measures the uniformity of the solutions [40].

$$
S P C=\frac{\sum_{i=1}^{\left|F_{1}\right|-1}\left|\bar{d}-d_{i}\right|}{\left(\left|F_{1}\right|-1\right) \times \bar{d}}
$$

Construction of the Pareto fronts in the MO-JSSP needs high computational effort, especially in the large-size problems, which have more than 20 jobs and 10 machines. So, achieving the Pareto front in a short time and with a small memory demonstrates the efficiency of the algorithm. Suresh and Mohanasundaram have reported that they have achieved a good solution set in a short computational time and with a small memory [38]. They have used the net front contribution ratio (NFCR) metric to measure the quality of the non-dominated solution set [29]. NFCR is a relative measure that uses the net non-dominated front as the reference set. The net non-dominated front is obtained by updating the combined non-dominated front, which consists of the all non-dominated solutions of the compared algorithms. $F_{i}$ stands for the non-dominated front of the $i$-th algorithm, while $F_{n}$ is the front of all algorithms. $n_{i}$ and $n_{n}$ are respectively the numbers of solutions in the non-dominated individuals of the $i$-th algorithm and the net front $\left(F_{n}\right)$. For each of the compared algorithm, the net front contribution ratio is calculated according to Equation 9 [24]. 


$$
N F C R_{i}=\frac{n_{i}}{n_{n}}
$$

\section{Algorithm and Implementation}

The general steps of the NSGA-II algorithm are summarized in Figure 2, which are similar to the M1-NSGA-II and M2NSGA-II.

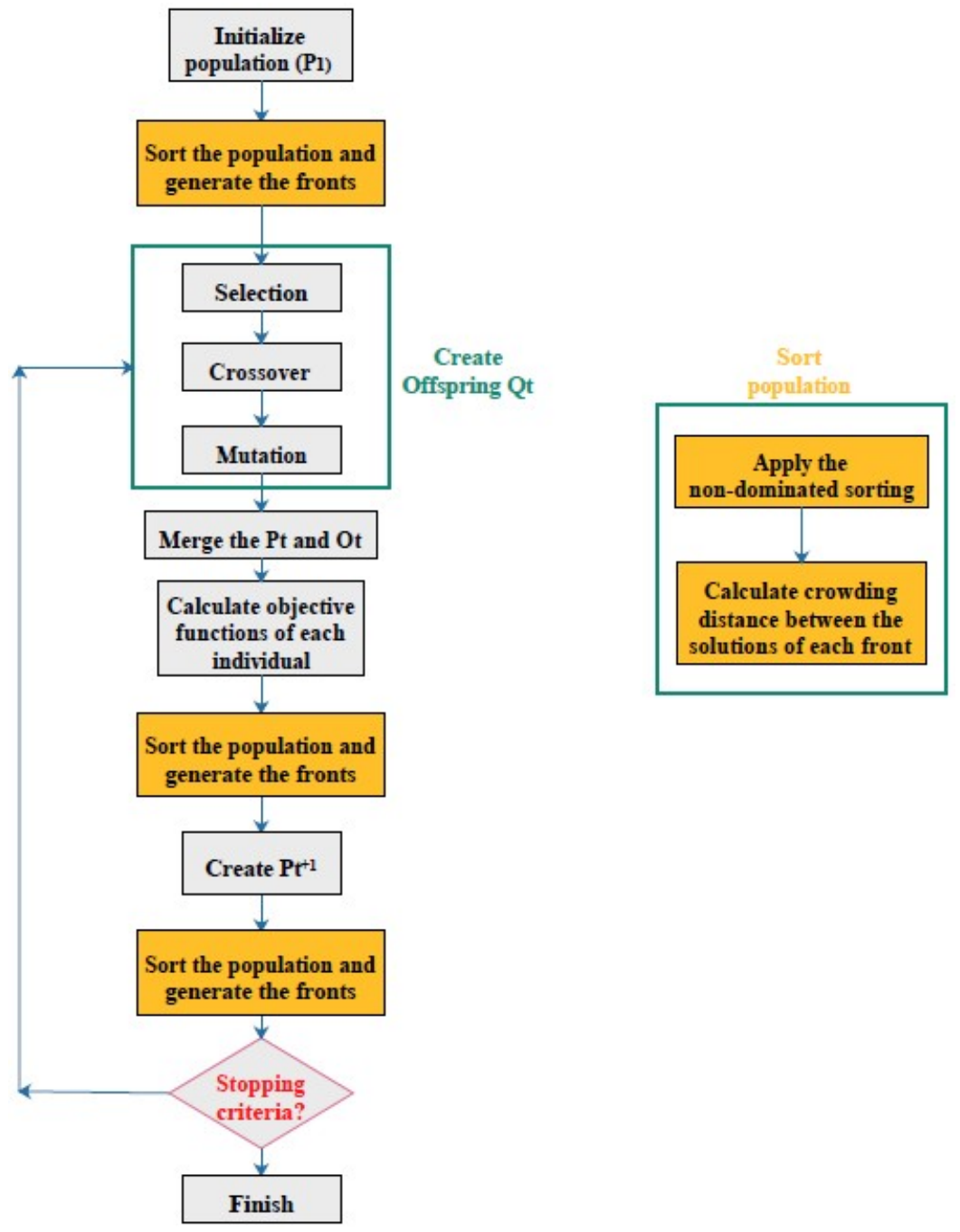

Figure 2. Flowchart of the NSGA-II

In the NSGA-II, the initial population is generated randomly, while in the M1-NSGA-II and M2-NSGA-II, $84 \%$ of the initial population is generated by the Giffler and Thompson (GT) algorithm [43] and the remainder part is created randomly. Each solution is expressed as a chromosome, which consists of two parts. As seen in Figure 3, the first part represents the order of operations, while the second part shows the assigned machines.

\begin{tabular}{|c|c|c|c|c|c|c|c|c|c|}
\hline $\mathrm{O}_{1}$ & $\mathrm{O}_{4}$ & $\mathrm{O}_{5}$ & $\mathrm{O}_{6}$ & $\mathrm{O}_{7}$ & $\mathrm{O}_{2}$ & $\mathrm{O}_{8}$ & $\mathrm{O}_{9}$ & $\mathrm{O}_{3}$ & $\mathrm{O}_{10}$ \\
\hline $\mathrm{M}_{1}$ & $\mathrm{M}_{2}$ & $\mathrm{M}_{3}$ & $\mathrm{M}_{1}$ & $\mathrm{M}_{3}$ & $\mathrm{M}_{2}$ & $\mathrm{M}_{1}$ & $\mathrm{M}_{3}$ & $\mathrm{M}_{2}$ & $\mathrm{M}_{3}$ \\
\hline
\end{tabular}

Figure 3. A chromosome that consists of two parts to represent the order of operations and the assigned machines

The initial population is sorted and its fronts are formed according to the non-dominated sorting and calculation of crowding distances, which are explained in Algorithms 1 and 2 and Figure 4. To construct the offspring population $\left(Q_{t}\right)$, the parents 
are selected through the tournament selection. For this purpose, if a solution is dominated, it is eliminated. If none of them is dominated by the other, the one with the better crowding distance is chosen as the parent. Crossover is defined as a onepoint operator that is applied to the first part. The mutation is done with a constraint-based neural network that creates feasible neighbors. It applies penalties to infeasible neighbors and transforms them to feasible ones. In this way, during the mutation, a feasible neighbor is generated for the selected individuals, according to the structures summarized in Algorithm 3.
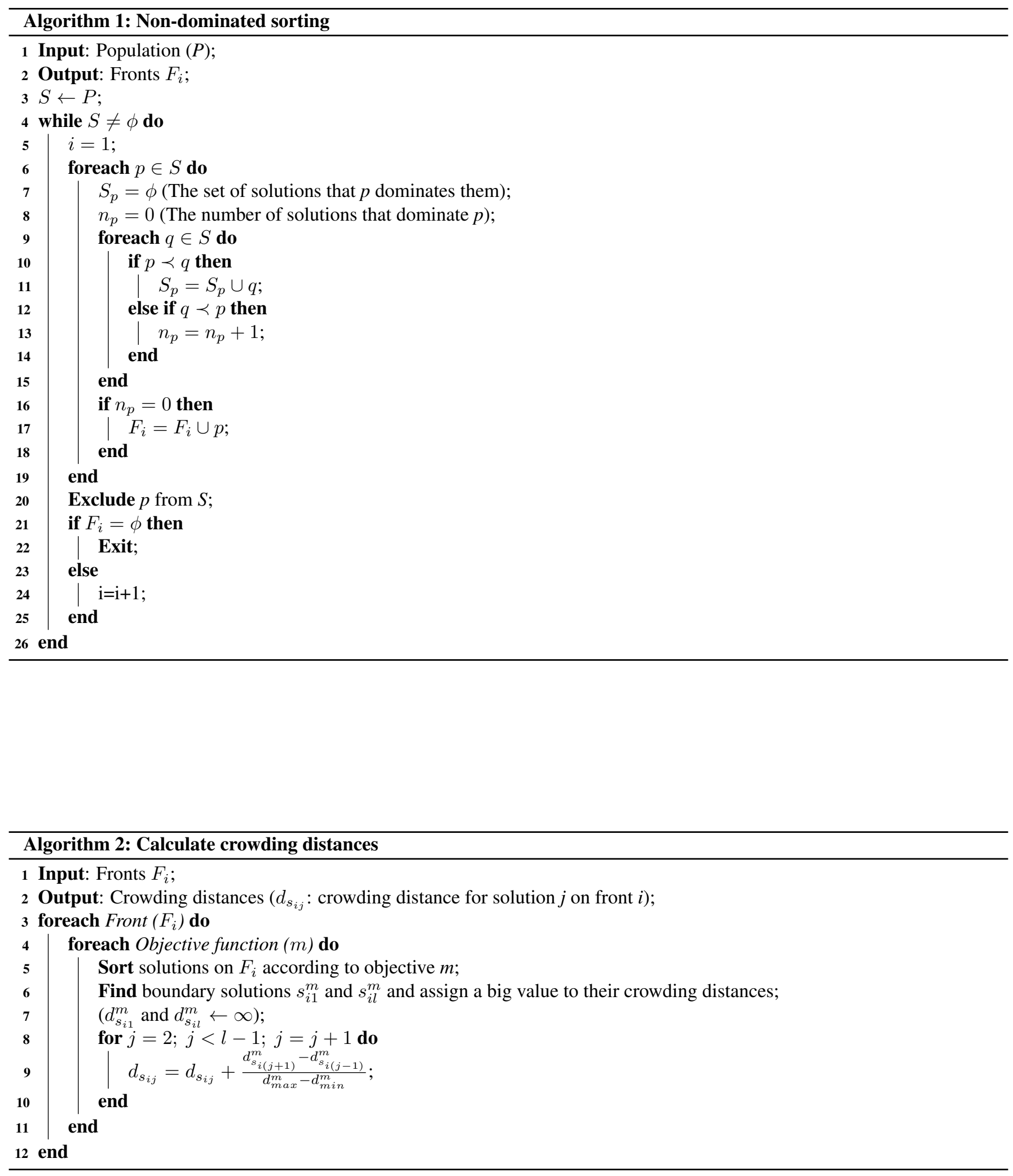


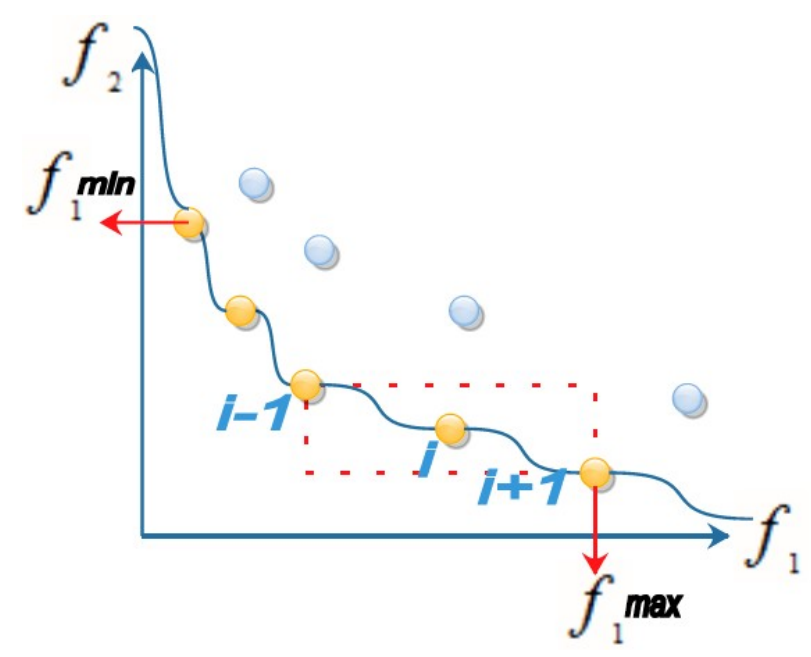

Figure 4. Crowding distance between to solutions

As seen in Figure 5, in the NSGA-II, the $Q_{t}$ is merged with the parent population $\left(P_{t}\right)$.

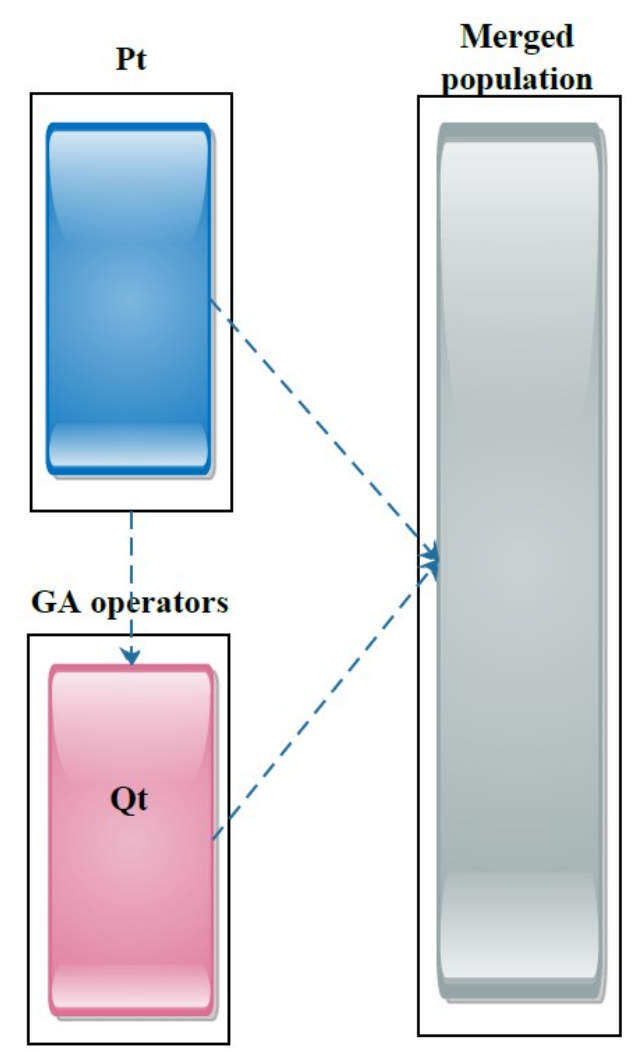

Figure 5. The creation of the merged population in the NSGA-II

In the M1-NSGA-II, as shown in Figure 6, after that the $Q_{t}$ is generated using the crossover and mutation operators, a neighbor is constructed for each individual using a neighborhood structure selected randomly from the structures that are summarized in Algorithm 3. If the neighbor dominates the parent, it is replaced with the parent. Else if the parent dominates the neighbor it reminds in the population. If they do not dominate the other, one of them is selected randomly, then the $Q_{t}$ is merged with the new $P_{t}$. 


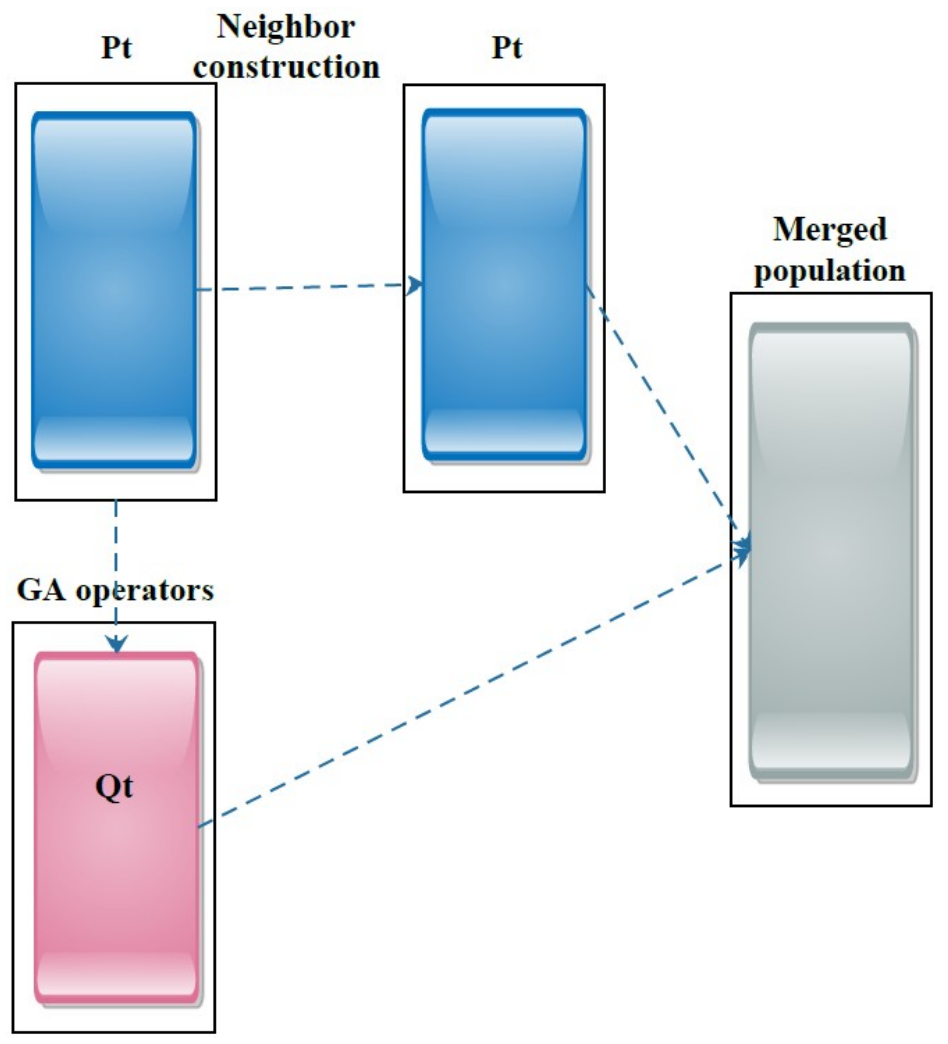

Figure 6. The construction of the merged population in the M1-NSGA-II

As seen in Figure 7, in the M2-NSGA-II, after creation of the $Q_{t}$ using the crossover and mutation operators, a neighbor is created only for the solutions on the first and second fronts of the $P_{t}$, using a neighborhood structure selected randomly from Algorithm 3. Then they are merged with the $Q_{t}$.

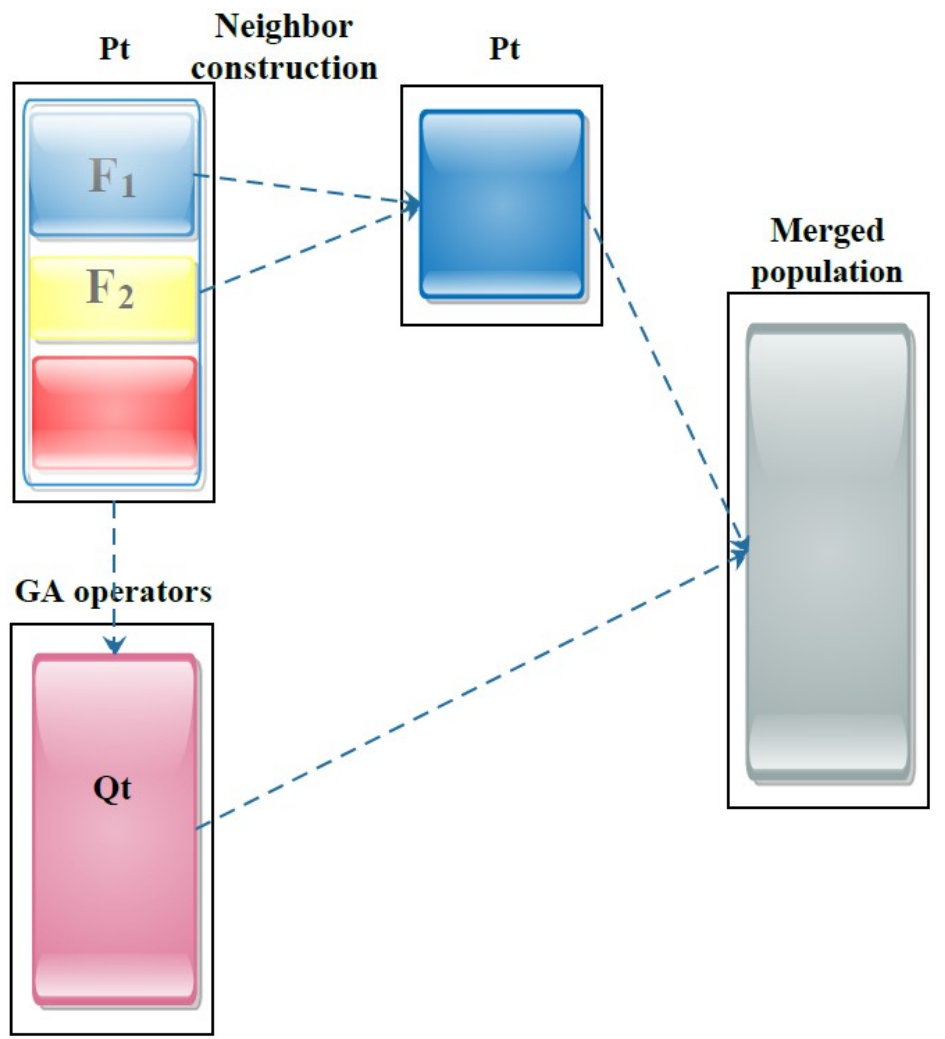

Figure 7. The generation of the merged population in the M2-NSGA-II 
The used neighborhood structures are summarized in Algorithm 3.

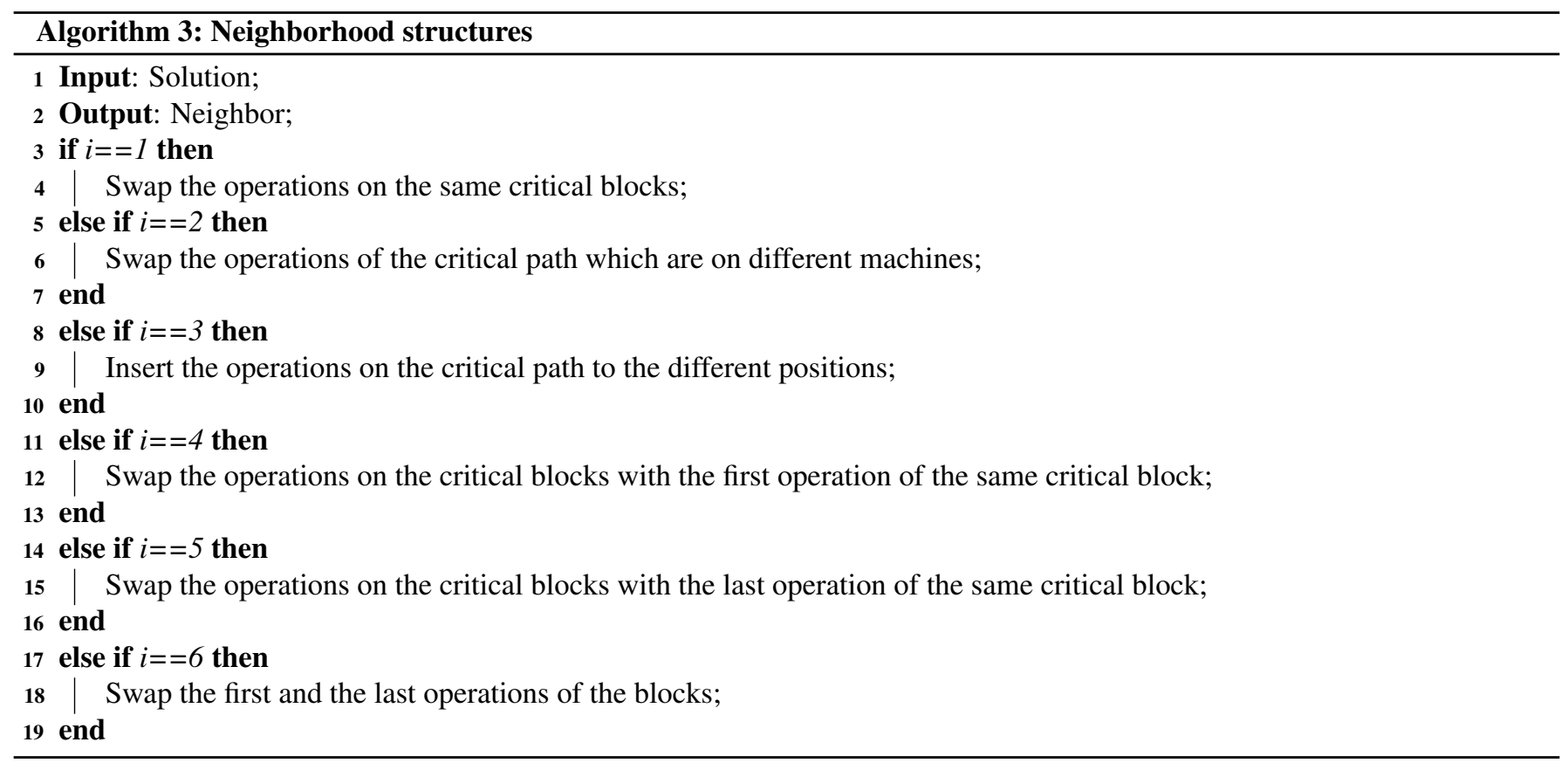

In the all algorithms, the merged population is sorted using the non-dominated sorting and crowding distances, then the new population $\left(P_{t+1}\right)$ is created with selecting solutions as much as the population size. These processes are summarized in Figure 8.

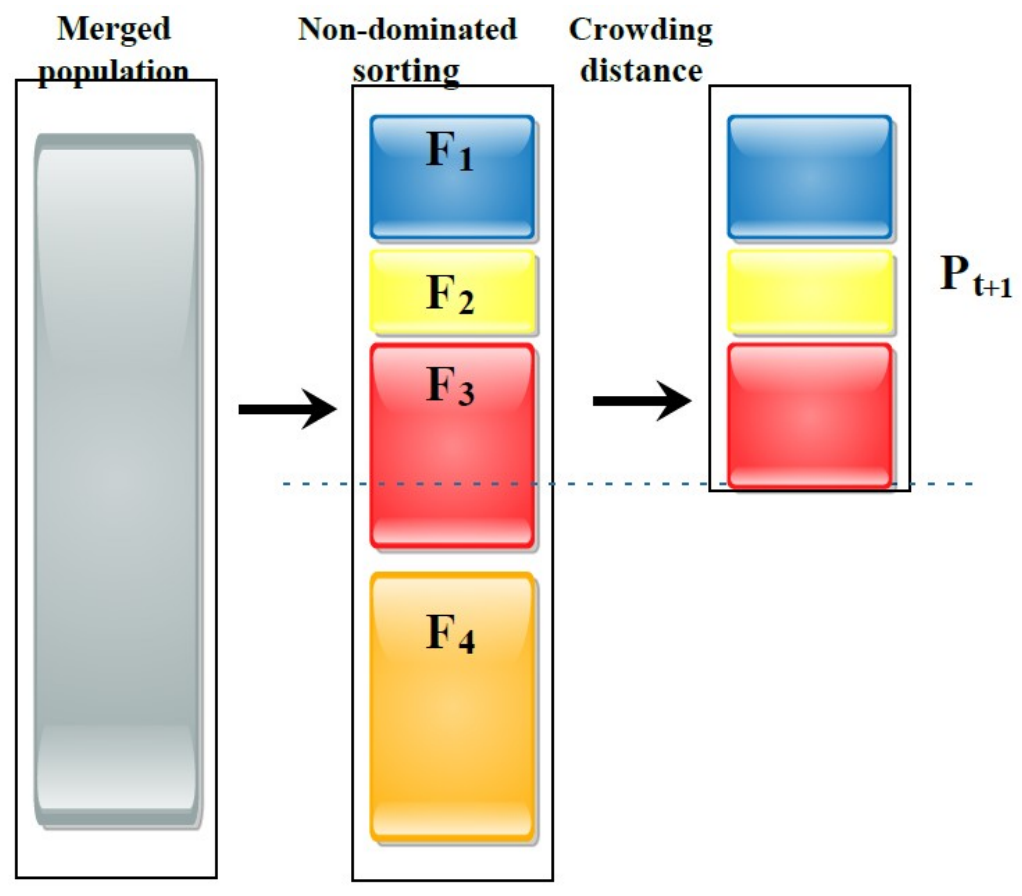

Figure 8. New population generation in the algorithms

\section{Results and Comparison}

The parameters of the designed algorithm are shown in Table 2. 
Table 2. Parameters of the proposed M-NSGA-II

\begin{tabular}{lc}
\hline Parameter & Value \\
\hline \hline Population size & 50 \\
\hline Crossover rate & $100 \%$ \\
\hline Mutation rate & $20 \%$ \\
\hline Number of iterations & 100 \\
\hline
\end{tabular}

The MK01 from the BRData instances is used as the benchmark, whose best intervals in literature are summarized in Table $3[25]$.

Table 3. Best intervals of the objective functions in the literature for the MK01 instance [25] (NS: Number of non-dominated solutions in the literature)

\begin{tabular}{|c|c|c|c|}
\hline$C_{\max }$ & $W_{m}$ & $W_{T}$ & $\mathrm{NS}$ \\
\hline$(40,45)$ & $(36,42)$ & $(153,169)$ & 11 \\
\hline
\end{tabular}

The described algorithms are implemented in MATLAB 2017b on a system with a Core i7 6700HQ processor and 32 GB RAM. The obtained results are summarized in Table 4. The NSGA-II, M1-NSGA-II and M2-NSGA-II have found 2, 2 and 1 Pareto solutions, respectively. The order of operations and the assigned machines in these solutions are presented in the appendix.

Table 4. The obtained results

\begin{tabular}{||c|c|c|c|c|c|c|c|c||}
\hline \multicolumn{3}{|c|}{ NSGA-II } & \multicolumn{3}{c|}{ M1-NSGA-II } & \multicolumn{3}{c|}{ M2-NSGA-II } \\
\hline$C_{\max }$ & $W_{m}$ & $W_{T}$ & $C_{\max }$ & $W_{m}$ & $W_{T}$ & $C_{\max }$ & $W_{m}$ & $W_{T}$ \\
\hline 63 & 60 & 196 & 53 & 48 & 178 & 62 & 54 & 197 \\
\hline 81 & 81 & 191 & 50 & 49 & 168 & & & \\
\hline
\end{tabular}

The comparison between the algorithms based on the measures $F O$ and $N F C R$, defined in Equations 4 and 9, is summarized in Table 5. The solutions achieved by the M1-NSGA-II dominate the other ones obtained by the NSGA-II and M2-NSGA-II algorithms. Therefore, its NFCR value is equal to 1 . None of the solutions obtained by the other 2 algorithms dominate the others, so their NFCR values are equal to 0 .

Table 5. The comparison based on the measures $F O$ and $N F C R$

\begin{tabular}{|c||c|c|c|}
\cline { 2 - 4 } \multicolumn{1}{c|}{} & NSGA-II & M1-NSGA-II & M2-NSGA-II \\
\hline$F O$ & 2 & 2 & 1 \\
\hline$N F C R$ & 0 & 1 & 0 \\
\hline
\end{tabular}

\section{Conclusion and Future Works}

In this study, the neighborhood structures defined for the FJSSP are integrated into the NSGA-II algorithm to increase the diversity and intensity of the search. In this way, the M1-NSGA-II and M2-NSGA-II algorithms are designed. During the iterations and before the merge of parent and offspring populations, the algorithms attempt to improve the search by finding the neighbors of some individuals. In the algorithms, the mutation is done by a constraint-based neural network that creates feasible neighbors. The algorithms are compared with a classic NSGA-II algorithm. The results show that the M1-NSGA-II, which creates neighbor for more individuals, has better performance than the others. Accordingly, it can be deduced that the use of neighborhood structures improves the performance of multi-objective optimization algorithms. Also, better results are obtained 
when heuristics such as the Giffler and Thompson algorithm and the local left shifts are used for initial solutions creation, and even to improve the solutions during the iterations. Based on the results obtained from the M2-NSGA-II, we can make another conclusion that it is better to define that the neighborhood structures should be applied to how many solutions and which ones. Otherwise, the performance of the algorithm may not improve.

In future work, we plan to design a multi-objective particle swarm optimization (MO-PSO) algorithm for solving scheduling problems.

\section{REFERENCES}

[1] O. Bahadir, G. Ozturk, and A. Teymourifar. Extracting new dispatching rules for dynamic multi-objective scheduling problems using simulation and gep. In 3 rd INTERNATIONAL RESEARCHERS, STATISTICIANS AND YOUNG STATISTICIANS CONGRESS 24-26 MAY 2017 SELÇUK UNIVERSITY, 2017.

[2] J. Behnamian, M. Zandieh, and S. F. Ghomi. Parallel-machine scheduling problems with sequence-dependent setup times using an aco, sa and vns hybrid algorithm. Expert Systems with Applications, 36(6):9637-9644, 2009.

[3] D. Behnke and M. J. Geiger. Test instances for the flexible job shop scheduling problem with work centers. https: //www.deutsche-digitale-bibliothek.de/item/WG35B3KZZC4YAARHH3GSHGXEKWRZHY5L.

[4] D. Behnke and M. J. Geiger. Test instances for the flexible job shop scheduling problem with work centers. 2012.

[5] J. H. Blackstone, D. T. Phillips, and G. L. Hogg. A state-of-the-art survey of dispatching rules for manufacturing job shop operations. The International Journal of Production Research, 20(1):27-45, 1982.

[6] J. Błażewicz, W. Domschke, and E. Pesch. The job shop scheduling problem: Conventional and new solution techniques. European journal of operational research, 93(1):1-33, 1996.

[7] P. Brandimarte. Routing and scheduling in a flexible job shop by tabu search. Annals of Operations research, 41(3):157183, 1993.

[8] J. B. Chambers and J. W. Barnes. Tabu search for the flexible-routing job shop problem. Graduate program in Operations Research and Industrial Engineering, The University of Texas at Austin, Technical Report Series, ORP96-10, 1996.

[9] G. C. Ciro, F. Dugardin, F. Yalaoui, and R. Kelly. A nsga-ii and nsga-iii comparison for solving an open shop scheduling problem with resource constraints. IFAC-PapersOnLine, 49(12):1272-1277, 2016.

[10] C. A. C. Coello, G. B. Lamont, D. A. Van Veldhuizen, et al. Evolutionary algorithms for solving multi-objective problems, volume 5. Springer, 2007.

[11] S. Dauzère-Pérès and J. Paulli. Solving the general multiprocessor job-shop scheduling problem. Working Papers, Department of Mathematical Sciences, University of Aarhus, 1995.

[12] S. Dauzère-Pérès and J. Paulli. An integrated approach for modeling and solving the general multiprocessor job-shop scheduling problem using tabu search. Annals of Operations Research, 70:281-306, 1997.

[13] K. Deb, S. Agrawal, A. Pratap, and T. Meyarivan. A fast elitist non-dominated sorting genetic algorithm for multi-objective optimization: Nsga-ii. In Parallel problem solving from nature PPSN VI, pages 849-858. Springer, 2000.

[14] K. Deb and H. Jain. An evolutionary many-objective optimization algorithm using reference-point-based nondominated sorting approach, part i: Solving problems with box constraints. IEEE Trans. Evolutionary Computation, 18(4):577-601, 2014.

[15] K. Deb, A. Pratap, S. Agarwal, and T. Meyarivan. A fast and elitist multiobjective genetic algorithm: Nsga-ii. Evolutionary Computation, IEEE Transactions on, 6(2):182-197, 2002.

[16] M. Dell'Amico and M. Trubian. Applying tabu search to the job-shop scheduling problem. Annals of Operations Research, 41(3):231-252, 1993.

[17] P. Fattahi, M. S. Mehrabad, and F. Jolai. Mathematical modeling and heuristic approaches to flexible job shop scheduling problems. Journal of Intelligent Manufacturing, 18(3):331-342, 2007.

[18] L. Gambardella and M. Mastrolilli. Effective neighborhood functions for the flexible job shop problem. Journal of scheduling, 3(3), 1996. 
[19] N. B. Ho, J. C. Tay, and E. M.-K. Lai. An effective architecture for learning and evolving flexible job-shop schedules. European Journal of Operational Research, 179(2):316-333, 2007.

[20] O. Holthaus and C. Rajendran. Efficient dispatching rules for scheduling in a job shop. International Journal of Production Economics, 48(1):87-105, 1997.

[21] T. Hsu, R. Dupas, D. Jolly, and G. Goncalves. Evaluation of mutation heuristics for solving a multiobjective flexible job shop by an evolutionary algorithm. In Systems, Man and Cybernetics, 2002 IEEE International Conference on, volume 5, pages 6-pp. IEEE, 2002.

[22] J. Hurink, B. Jurisch, and M. Thole. Tabu search for the job-shop scheduling problem with multi-purpose machines. Operations-Research-Spektrum, 15(4):205-215, 1994.

[23] H. Ishibuchi and T. Murata. A multi-objective genetic local search algorithm and its application to flowshop scheduling. Systems, Man, and Cybernetics, Part C: Applications and Reviews, IEEE Transactions on, 28(3):392-403, 1998.

[24] H. Ishibuchi, T. Yoshida, and T. Murata. Balance between genetic search and local search in memetic algorithms for multiobjective permutation flowshop scheduling. IEEE transactions on evolutionary computation, 7(2):204-223, 2003.

[25] S. Jia and Z.-H. Hu. Path-relinking tabu search for the multi-objective flexible job shop scheduling problem. Computers \& Operations Research, 47:11-26, 2014.

[26] I. Kacem, S. Hammadi, and P. Borne. Approach by localization and multiobjective evolutionary optimization for flexible job-shop scheduling problems. Systems, Man, and Cybernetics, Part C: Applications and Reviews, IEEE Transactions on, 32(1):1-13, 2002.

[27] I. Kacem, S. Hammadi, and P. Borne. Pareto-optimality approach for flexible job-shop scheduling problems: hybridization of evolutionary algorithms and fuzzy logic. Mathematics and computers in simulation, 60(3):245-276, 2002.

[28] J. Knowles and D. Corne. The pareto archived evolution strategy: A new baseline algorithm for pareto multiobjective optimisation. In Evolutionary Computation, 1999. CEC 99. Proceedings of the 1999 Congress on, volume 1, pages 98-105. IEEE, 1999.

[29] J. D. Knowles. Local-search and hybrid evolutionary algorithms for Pareto optimization. PhD thesis, Department of Computer Science Local-Search and Hybrid Evolutionary Algorithms for Pareto OptimiZation Joshua D. Knowles Submitted in partial fulfilment of the requirements for the degree of Doctor of Philosophy in Computer Science. Department of Computer Science, University of Reading, 2002.

[30] F. Kursawe. A variant of evolution strategies for vector optimization. In Parallel Problem Solving from Nature, pages 193-197. Springer, 1990.

[31] Y.-W. Leung and Y. Wang. Multiobjective programming using uniform design and genetic algorithm. Systems, Man, and Cybernetics, Part C: Applications and Reviews, IEEE Transactions on, 30(3):293-304, 2000.

[32] H. Nakayama, K. Kaneshige, S. Takemoto, and Y. Watada. An application of a multi-objective programming technique to construction accuracy control of cable-stayed bridges. European Journal of Operational Research, 87(3):731-738, 1995.

[33] E. Nowicki and C. Smutnicki. A fast taboo search algorithm for the job shop problem. Management science, 42(6):797-813, 1996.

[34] V. Roshanaei, B. Naderi, F. Jolai, and M. Khalili. A variable neighborhood search for job shop scheduling with set-up times to minimize makespan. Future Generation Computer Systems, 25(6):654-661, 2009.

[35] G. Rudolph. Evolutionary search under partially ordered sets. Dept. Comput. Sci./LS11, Univ. Dortmund, Dortmund, Germany, Tech. Rep. CI-67/99, 1999.

[36] C. Santos, J. Spim, and A. Garcia. Mathematical modeling and optimization strategies (genetic algorithm and knowledge base) applied to the continuous casting of steel. Engineering Applications of Artificial Intelligence, 16(5):511-527, 2003.

[37] J. Schafer. Multi-objective optimization with vector evaluated genetic algorithm. In Proceedings of the 1st International Conference on Genetic Algorithms, pages 93-100.

[38] R. Suresh and K. Mohanasundaram. Pareto archived simulated annealing for job shop scheduling with multiple objectives. The International Journal of Advanced Manufacturing Technology, 29(1-2):184-196, 2006. 
[39] E.-G. Talbi. Metaheuristics: from design to implementation, volume 74. John Wiley \& Sons, 2009.

[40] R. Tavakkoli-Moghaddam, M. Azarkish, and A. Sadeghnejad-Barkousaraie. A new hybrid multi-objective pareto archive pso algorithm for a bi-objective job shop scheduling problem. Expert Systems with Applications, 38(9):10812-10821, 2011.

[41] A. Temourifar and G. Ozturk. New dispatching rules and due date assignment models for job shop scheduling problems. Accepted to International Journal of Manufacturing Research, 2017.

[42] A. Teymourifar and G. Ozturk. A hybrid pareto-based swarm optimization algorithm for the multi-objective flexible job shop scheduling problems. World Academy of Science, Engineering and Technology, International Journal of Mechanical and Industrial Engineering, 4(7), 2017.

[43] A. Teymourifar and G. Ozturk. A neural network-based hybrid method to generate feasible neighbors for flexible job shop scheduling problem. Universal Journal of Applied Mathematics, 6(1):1-16, 2018.

[44] A. Teymourifar, G. Ozturk, and O. Bahadir. A constrained neural network based variable neighborhood search for the multi-objective dynamic flexible job shop scheduling problems. World Academy of Science, Engineering and Technology, International Journal of Mechanical and Industrial Engineering, 4(7), 2017.

[45] B.-O. Teymourifar, Aydin and G. Ozturk. Dynamic priority rule selection for solving multi-objective job shop scheduling problems. Universal Journal of Industrial and Business Management, 6(1):11-22, 2018.

[46] L.-F. Tung, L. Lin, and R. Nagi. Multiple-objective scheduling for the hierarchical control of flexible manufacturing systems. International Journal of Flexible Manufacturing Systems, 11(4):379-409, 1999.

[47] P. Vachhani, R. Rengaswamy, and V. Venkatasubramanian. A framework for integrating diagnostic knowledge with nonlinear optimization for data reconciliation and parameter estimation in dynamic systems. Chemical Engineering Science, 56(6):2133-2148, 2001.

[48] W. Xia and Z. Wu. An effective hybrid optimization approach for multi-objective flexible job-shop scheduling problems. Computers \& Industrial Engineering, 48(2):409-425, 2005.

[49] L.-N. Xing, Y.-W. Chen, P. Wang, Q.-S. Zhao, and J. Xiong. A knowledge-based ant colony optimization for flexible job shop scheduling problems. Applied Soft Computing, 10(3):888-896, 2010.

[50] L.-N. Xing, Y.-W. Chen, and K.-W. Yang. An efficient search method for multi-objective flexible job shop scheduling problems. Journal of Intelligent Manufacturing, 20(3):283-293, 2009.

[51] Y. Yuan and H. Xu. Multiobjective flexible job shop scheduling using memetic algorithms. Automation Science and Engineering, IEEE Transactions on, 12(1):336-353, 2015.

[52] G. Zhang, X. Shao, P. Li, and L. Gao. An effective hybrid particle swarm optimization algorithm for multi-objective flexible job-shop scheduling problem. Computers \& Industrial Engineering, 56(4):1309-1318, 2009.

[53] E. Zitzler. Evolutionary algorithms for multiobjective optimization: Methods and applications, volume 63. Citeseer, 1999.

[54] E. Zitzler, K. Deb, and L. Thiele. Comparison of multiobjective evolutionary algorithms: Empirical results. Evolutionary computation, 8(2):173-195, 2000. 


\section{Appendices}

The achieved solutions by the NSGA-II $\left(S_{1}, S_{2}\right)$, the M1-NSGA-II $\left(S_{3}, S_{4}\right)$ and the M2-NSGA-II $\left(S_{5}\right)$ are as follows:

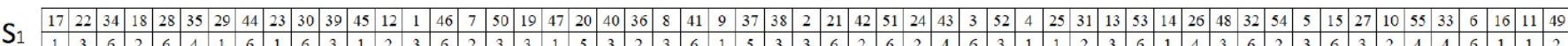

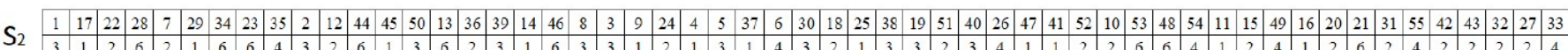

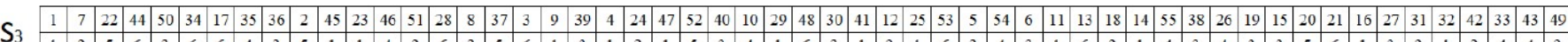

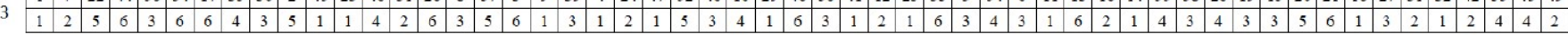

\begin{tabular}{|l|l|l|l|l|l|l|l|l|l|l|l|l|l|l|l|l|l|l|l|l|l|l|l|l|l|l|l|l|l|l|l|l|l|l|l|l|l|l|l|l|l|l|l|l|l|l|l|l|l|l|l|l|l|l|l|l|}
\hline $\mathrm{S}_{4}$ & 12 & 17 & 22 & 34 & 50 & 1 & 28 & 35 & 39 & 44 & 2 & 13 & 29 & 40 & 18 & 45 & 23 & 46 & 14 & 51 & 3 & 41 & 7 & 4 & 19 & 52 & 30 & 47 & 20 & 53 & 54 & 31 & 48 & 21 & 15 & 8 & 5 & 55 & 24 & 32 & 36 & 9 & 33 & 6 & 16 & 37 & 38 & 25 & 42 & 26 & 49 & 27 & 43 & 10 & 11 \\
\hline
\end{tabular}

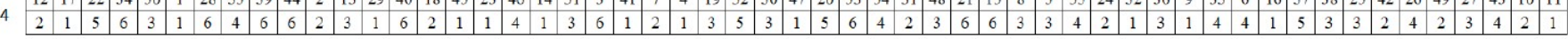

\begin{tabular}{|l|l|l|l|l|l|l|l|l|l|l|l|l|l|l|l|l|l|l|l|l|l|l|l|l|l|l|l|l|l|l|l|l|l|l|l|l|l|l|l|l|l|l|l|l|l|l|l|l|l|l|l|l|l|l|l|}
\hline$S_{5}$ & 1 & 12 & 17 & 22 & 39 & 28 & 2 & 23 & 40 & 18 & 3 & 29 & 30 & 41 & 4 & 19 & 24 & 20 & 50 & 13 & 51 & 7 & 14 & 21 & 25 & 5 & 15 & 52 & 8 & 9 & 26 & 6 & 16 & 10 & 27 & 42 & 44 & 45 & 53 & 11 & 46 & 31 & 47 & 54 & 48 & 32 & 43 & 33 & 55 & 34 & 49 & 35 & 36 & 37 & 38 \\
\hline
\end{tabular}

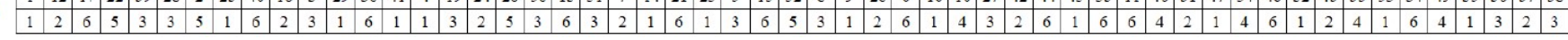

Figure 9. The obtained solutions 